\title{
Effects of Immersive Media on Emotion and Memory: An Experiment Comparing Article, 360-video, and Virtual Reality
}

\author{
Mila Bujić a,b \\ Mikko Salminen ${ }^{\mathrm{a}}$ \\ mila.bujic@tuni.fi \\ mikko.salminen@iki.fi \\ Juho Hamari ${ }^{\mathrm{a}}$ \\ juho.hamari@tuni.fi \\ ${ }^{a}$ Gamification Group, Faculty of Information Technology and Communication Sciences, Tampere University \\ ${ }^{b}$ Corresponding Author
}

\section{Abstract}

Fields such as immersive journalism and VR-based media consumption are expected to holistically engross users and have a deeper emotional impact as well as internalisation of information. This betweensubjects experiment $(\mathrm{N}=87)$ employs a 360-degree video was presented either on mobile VR, on screen, or as a written article consisting of the transcript and video stills. The results suggest that the higher immersiveness has a more prominent effect on the emotional response and higher negative affect to predict lower cued recall memory of the presented content. Age in the range does not seem to influence the strength of the affect change but females might experience a higher change than males. This study suggest caution when creating highly emotional content. The negative emotional experience might impede memory of factual information. These implications are further applicable to other related fields, such as education and simulation trainings.

Keywords: Immersive journalism; affect; emotional valence; memory; cued recall; 360-degree video

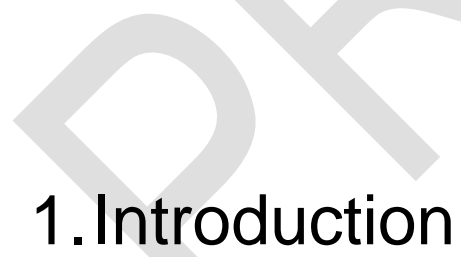

The past decade in media research has been marked by the drive and development of immersive, motivational, and persuasive media. For example, gamification (Koivisto and Hamari 2019), immersive virtual reality (Bailenson 2018), and persuasive games (Bogost 2007, Jacobs 2020) have all been increasingly popular and are changing the ways we learn, train skills, and play. This trend has recently seeped more noticeably into the sphere of journalism by utilising immersive media in attempts to convey content in a more engrossing way both in terms of emotion and information (de la Peña 2010, for a review see Bujić and Hamari 2020b). Immersive 
journalism has received significant attention in the recent years with the VR technologies and contents reaching more users than ever before (Bujić and Hamari 2020b). A distinct driver has been the release of various mobile VR headsets that, although not as high-end as fully immersive VR, instigated the production of 360-degree videos by large news houses (Baía Reis and Coelho 2018). This engrossing placement of the user in another's shoes in fully immersive VR has received wide public acknowledgement when such a piece received the special Oscar award in 2017 (Raessens 2019). These developments towards empathy-inducing experiences open up a range of possibilities with the touted potential of building a more inclusive world (Milk 2015, Bujić et al. 2020, Sánchez Laws 2020, Shin 2018).

While the pursuit for increasing empathy towards others through immersive journalism and media is a virtuous endeavor, there is a prior need of answering basic questions about the media effects that precede the formation of empathy and consequent changes in attitudes and behaviors. Media effects classically and in their roots categorize into those of cognitive (i.e. what we remember of the content) and affective (i.e. how does the content affect our emotional state). These touted gains in empathy and attitude change towards others from immersive journalism rely on these basic reactions to the content. For example, empathy is viewed as a synchronisation of affective states whereas it is achieved through the mechanism of emotional contagion, or experiencing by oneself emotions that are observed in another (de Vignemont 2006, Elfenbein 2014, Salminen et al 2018). Therefore, considering the effects on emotional states, whose operationalisation has been very well established (Watson and Clark 1988), provides a more reliable gateway to consider the effects of immersive journalism.

However, this potential of immersive media and immersive journalism in particular to affect one's emotions is not without its caveats. One of the main issues is the ethics of this field (e.g. Sánchez Laws and Utne 2019) considering, for example, possible psychological harm and the appropriate handling of sensitive topics. Additionally, it has been rightfully noted that this kind of media that specifically targets one's own experiential affect can easily borderline propaganda (Kool 2016). Although journalism has never been devoid of ideology and propaganda, the use of such persuasive and immersive means as through virtual reality, that specifically targets users' affective experiences, might exacerbate the problem and further blur this fine line (Kool 2016, Matthes 2013). In the wider context, the perils of fake news (Martel, Pennycook and Rand 2020) and the struggles in raising global media literacy (Potter 2010), prompt caution for balancing the need for conveying information on one side and eliciting affective persuasive experiences on the other.

The study of media effects of immersive journalism could contribute to the endeavor of building societal resilience towards detrimental effects, such as spreading of misinformation. As a contribution to the discussion on effects and implications of immersive journalism and immersive media in general, this study addresses the gap by investigating how does immersive journalism influence users' affective and cognitive processes when compared to a written article 
as a traditional journalism form? The research question is answered by examining three distinctive issues: 1) the effects of different media types on negative and positive affect, 2) the effects of media types on users' memory of their contents, and 3) the effects of negative affect on the memory of the presented contents.

\section{Background and hypotheses}

Previous studies on affective and cognitive media effects reveal two issues to consider. The first is that majority of studies are, appropriately, based on strictly controlled laboratory experiments and introduce extremely simplistic stimuli such as individual words or images (e.g. Bookbinder and Brainerd 2016, Gomes Brainerd and Stein 2013). Their results are therefore not directly transferrable to complex, immersive media environments and experiences. Second, the complexity also heightens when considering the effects of different dimensions of emotions (e.g. valence and arousal) and types of memory (e.g free recall and cued recall).

\subsection{Immersive media and emotional valence}

Considering that it has been repeatedly shown that even the simplest stimuli consisting of, for example, a single word can elicit an emotional reaction (Kensinger and Corkin 2003), it is to be assumed that all media forms are capable of evoking a change in the emotional state of the one reading, watching, or listening to the contents. The valence of user's emotions, positive or negative, is then expected to primarily depend on the content itself, as recently shown in virtual environments as well (e.g. Felnhofer 2015, Cadet and Chainay 2020). Therefore, in the case of media representing, for example, a personal refugee story conveyed through a first-person perspective, users are likely to experience heightened negative valence and lowered positive valence due to emotional contagion as the tendency of experiencing the emotions conveyed through media (Elfenbein 2014).

H1.1: Consuming sorrowful first-person media content elicits a lowered positive affect regardless of the media type.

H1.2: Consuming sorrowful first-person media content elicit a heightened negative affect regardless of the media type.

However, different narrative forms and media types such as film are more successful than others in eliciting a change in the emotional state due to different levels of emotional arousal, and different auditory stimuli affect one's emotions in particular ways (Swaminathan and Schellenberg 2015). These particularities are also seen to drive the change in the taste in media during emotionally turbulent adolescent and young adult years, as well as to explain the differences in which music appeals to males and females (Dibben 2002). Therefore, the extent of media effects (Valkenburg, Peter and Walther, 2016) on emotions may vary depending on at 
least two considerations: a) the type of media and media that is used for presenting a story; b) the individual differences of the users.

When it comes to media types, it has been suggested that those engaging a greater number of senses and so engulf, or immerse the consumer,it are likely to evoke a higher emotional response (Ding, Zhou and Fung 2018, Kim et al. 2018, Visch, Tan and Molenaar 2010). This is also consistent with the recent indication that the criteria for age ratings should be reconsidered for the context of immersive media (Wilson and McGill 2018). With new, immersive media, this could be explained primarily by the technological immersiveness of the content and media technology, such as virtual reality. The wider field of view, for example, enhances the feeling of non-mediation, which in turn enhances the experience, perceived personal closeness to the story, and finally the emotional experience.

H2.1: Higher immersiveness of media representing sorrowful media content predicts a higher decrease in the positive affect.

H2.2: Higher immersiveness of media representing sorrowful media content predicts a higher increase in the negative affect.

Individual differences of consumers shape how individuals perceive the content distinctive to their own personality and experiences. An important aspect relevant to immersive media with emotional content is the susceptibility to emotional contagion. It has been shown that gender is one of the influencing factors, with females consistently scoring higher on changes in emotions consistent with those observed in the stimuli (Doherty et al. 1995, Kevrekidis 2008). Therefore, it is expected that females would experience a stronger effect of the emotional media experience than males.

H3.1: Females experience a more prominent negative affect change compared to males.

It has been shown that typically younger adults are more susceptible to negativity bias and remember negative information more efficiently than neutral or positive information (Spaniol, Voss \& Grady, 2008). This heightened attention is closely connected to the lived experiences of emotional stimuli, with younger adults being more prone to heightened emotional reactions (Yuan et al. 2015, Birditt 2014).

H3.2: Higher age predicts a lower negative affect change.

\subsection{Immersive media, negative emotions and memory}

There is a rich body of literature indicating that negative content and consequent negative affect enhance memory, namely related to the negativity bias hypothesis and the theory of emotional enhancement of memory (EEM). Negativity bias hypothesis states that negative content is remembered better than positive material and experiences and that individuals gravitate towards 
focusing their attention on the negative aspects or content rather than positive (Robinson-Riegler and Winton 1996). Similarly, EEM posits that the strength of emotional responses positively predicts memory of the source events. This is likely due to higher emotional arousal, which regardless of the valence of the emotions enhances memory, as shown by Lang, Dillon and Dong (2009). However, the same study also suggested that when controlling for the arousal, positive messages were remembered better than the negative ones, which contradicts with the negativity bias hypothesis.

This might be due to the negativity bias hypothesis and EEM often not accounting for different types of memory. The fuzzy-trace theory (FTT) differentiates between verbatim and gist memory and the mechanisms that build them (Brainard and Reyna 2004). This distinction is particularly relevant for the context of immersive journalism for understanding the difference between remembering the overall story and one's experience of it on one side, and specific information presented in the materials on the other. It has been shown, for example, that when controlling for emotional arousal there is a decrease of verbatim true memories and an increase of false memories when the stimulus is negatively valenced (Lang, Dhillon, and Dong 1995, Van Damme and Smets 2014, Brainerd et al. 2008). On the other hand, recall of the gist is higher in both negatively and positively valenced stimuli, compared to neutral (Bookbinder and Brainerd 2017). Finally, in a study in the context of immersive media it was suggested that, compared to positive stimuli, negatively valenced stimuli elicits lower memory (Cadet and Chainay 2020).

These findings are not completely consistent nor directly applicable to the complex stimuli of 360-degree immersive journalism video content. However, they suggest that in the context of the present study the memory of the verbatim information could be impeded due to the negative valence of the stimulus. This is depicted in hypotheses H4.1 and, congruently with previous hypotheses, in H4.2. As the H2.2 predicts higher negative affect change in conditions with higher immersion, this effect would further translate into heightened effects on cued recall.

H4.1: Higher negative affect predicts lower cued recall.

H4.2: Higher media immersiveness predicts lower cued recall.

\section{Method}

The between-subjects design employed three conditions differing in their level of technological immersiveness, with Article being low $(\mathrm{n}=27), 2 \mathrm{D}$ medium $(\mathrm{n}=29)$, and VR high immersion $(\mathrm{n}=31)$. The sample size was determined under the heuristic of assigning approximately 30 participants per group and a smallest effect size expressed through Cohen's $d$ of about 0.3 (Lakens, 2021). The effect size was approximated considering that the interest of this study is in the potential practical effects of consuming an immersive journalism video. All statistical analyses were performed using IBM SPSS v25. 


\subsection{Participants}

Participants $(\mathrm{N}=87)$ were recruited via flyers and student email lists (Age $M=26.4, S D=4.5$ ), range: 18-40). Two-thirds were males and the sample was randomly distributed across the three conditions while minding the gender division. The sample is a convenience, non-probability sample recruited through various university mailing lists and information boards.

\subsection{Materials}

The content of the 360-video The Sea Prayer by Guardian VR was used as the stimulus. In the HMD and 2D conditions, the video was used in its original form and played from the Guardian $V R$ and YouTube respectively. In the Article condition, a transcript and video stills were compiled to resemble an Internet news article. The video length is 7:10 minutes and three scenes can be discerned in a final panoramic view.

The stimulus presents the story of a man who, after a peaceful life, found himself living in a conflict and war-struck area, and finally sitting on a beach with a baby boy in his arms, waiting for ships to emigrate (Figure 1). It is narrated in first-person and the prologue informs the viewer that the story is based on the case of Alan Kurdi, an emigrant boy who drowned and was found on a beach.

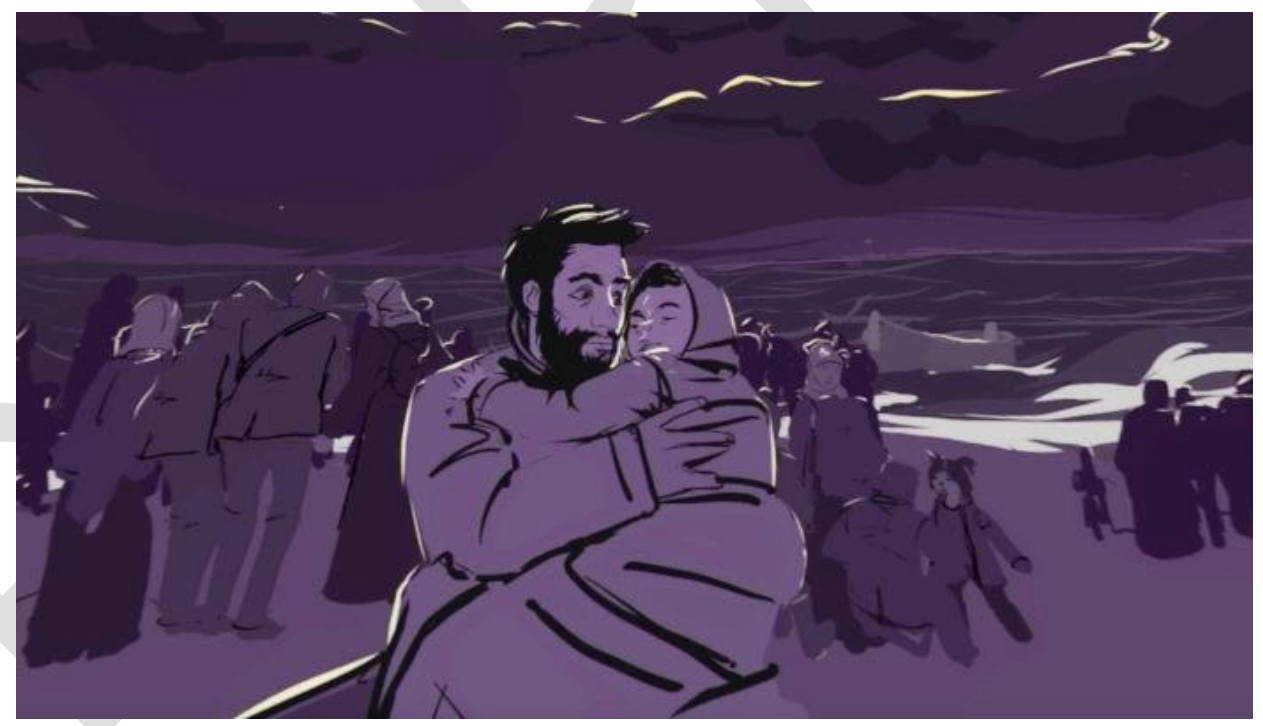

Figure 1. Third scene from The Sea Prayer by The Guardian VR.

\subsubsection{Positive and Negative Affect Schedule (PANAS)}

The emotional response was investigated using a pretest-posttest design. Participants' reported their Negative affect (NA) and Positive affect (PA) using 10 items for each, adopted from Watson, Clark and Tellegen (1988). As the sample consists predominantly of international 
students, one item (jittery) from NA was discarded prior to conducting the analyses due to some of the participants indicating that they did not understand its meaning. The instrument has been widely used and validated (Crawford and Henry 2004) and in this study, the scales' reliability was assessed using Cronbach's Alpha. For the NA dimension, the obtained values for the preand post-tests were .88 and .88 , whereas for PA they were .89 and .87 , all indicating very good scale reliability. The pretest was administered immediately before the treatment to establish a baseline and the posttest was administered immediately after the treatment to track the changes in the emotional states.

The scale was introduced as follows: "This scale consists of a number of words that describe different feelings and emotions. Read each item and then mark the appropriate answer on the scale. Indicate to what extent you feel this way right now, that is, at the present moment". Each emotion was marked on a 5-point Likert scale, with anchors worded as Very slightly or not at all and Extremely.

The baseline mean scores of PA and NA were deducted from the posttest ones, generating two variables presenting PA and NA changes that occurred during the treatment (i.e. PA = posttest $\mathrm{PA}$ - pretest PA). In the case of NA, this results in positive numbers representing higher negative valence after the treatment than before.

\subsubsection{Cued recall}

Memory was measured using 10 multiple choice questions about the content, constructed by the authors. The memory test was constructed so that it includes information presented in different ways (i.e. visual, explicit in narration, implicit in narration). All questions, answer options, and correct answers are presented in the Appendix. The order of questions and options were randomised. Answers were scored so that the correct choice is marked as 1 and the incorrect as 0 . The total was then summarized into a single variable to be used in the analyses, with the maximum value of 10 and minimum of 0 .

\subsection{Procedure}

The experiment was held at Tampere University premises, in a room with a desk, computer monitor, and a revolving office chair. Demographic details were collected a few days before attending the experiment at the same time when reserving a time slot for participation. Upon arrival, they first completed the PANAS questionnaire to establish a baseline of their affective states immediately before the treatment. Next, they received instructions depending on their random assignment to one of three conditions. The instructions pertained solely to how to operate the technology: for the Article condition, they involved stating that they will be presented with an article to read and that they can freely scroll through it as they see appropriate; for the 2D condition, an explanation of the click-and-drag mechanism of surveying the 360- 
degree panoramic video was given; for the VR condition, similar explanation was given but for the mobile VR version, where they were free to revolve on the chair and turn their head to left and right in order to survey the 360-degree video. Participants completed PANAS and cued recall questionnaires immediately after the designated treatment. Finally, they were informed in more detail about the design and aim of study and offered a contact for any further comments or questions.

Information regarding the aim of the study, procedure, anonymity protection, voluntary participation principles, and compensation of two movie vouchers was provided prior to collecting any data from the participants. The experiment was planned and conducted following the guidelines of the National Board on Research Integrity.

\section{Results}

The basic descriptive statistics are shown in Table 1. Furthermore, Table 2 presents Pearson's 2tailed inter-correlation coefficients between all measured variables that were used in the analyses. Already from the correlation matrix it is visible that participants' age did not have a relationship with any of the other variables, suggesting no support for H3.2.

\begin{tabular}{|c|c|c|c|c|}
\hline & Mean (SD) & Mean $95 \%$ CI & Median & Variance \\
\hline \multicolumn{5}{|l|}{$\begin{array}{l}\text { Pre PA } \\
\text { Post PA }\end{array}$} \\
\hline \multirow[t]{2}{*}{ Article } & $3.25(.62)$ & $3.00,3.49$ & 3.30 & .384 \\
\hline & $2.85(.83)$ & $2.52,3.18$ & 2.80 & .696 \\
\hline \multirow[t]{2}{*}{$2 \mathrm{D}$} & $3.19(.71)$ & $2.92,3.46$ & 3.10 & .500 \\
\hline & $2.85(.61)$ & $2.62,3.08$ & 3.00 & .373 \\
\hline \multirow[t]{2}{*}{ VR } & $3.05(.83)$ & $2.75,3.36$ & 3.10 & .687 \\
\hline & $2.72(.81)$ & $2.43,3.02$ & 2.80 & .655 \\
\hline \multicolumn{5}{|l|}{ Pre NA } \\
\hline \multicolumn{5}{|l|}{ Post NA } \\
\hline \multirow[t]{2}{*}{ Article } & $1.57(.77)$ & $1.27,1.88$ & 1.22 & .593 \\
\hline & $1.80(.76)$ & $1.50,2.10$ & 1.56 & .575 \\
\hline \multirow[t]{2}{*}{$2 \mathrm{D}$} & $1.61(.62)$ & $1.37,1.84$ & 1.33 & .386 \\
\hline & $2.17(.71)$ & $1.90,2.14$ & 2.11 & .506 \\
\hline \multirow[t]{2}{*}{ VR } & $1.50(.58)$ & $1.29,1.71$ & 1.33 & .332 \\
\hline & $2.09(.78)$ & $1.80,2.37$ & 1.89 & .603 \\
\hline \multicolumn{5}{|l|}{ PA Change } \\
\hline \multicolumn{5}{|l|}{ NA Change } \\
\hline \multirow[t]{2}{*}{ Article } & $-.40(.66)$ & $-.66,-.13$ & -.20 & .438 \\
\hline & $.23(.41)$ & $.07, .39$ & .11 & .167 \\
\hline \multirow[t]{2}{*}{$2 \mathrm{D}$} & $-.34(.62)$ & $-.57,-.10$ & -.40 & .387 \\
\hline & $.56(.74)$ & $.28, .84$ & .44 & .542 \\
\hline
\end{tabular}




\begin{tabular}{ccccc} 
VR & $-.33(.65)$ & $-.57,-.09$ & -.30 & .425 \\
& $.59(.60)$ & $.37, .81$ & .56 & .360 \\
\hline Memory & & & \\
\hline Article & $6.30(1.54)$ & $5.69,6.91$ & 6.00 & 2.370 \\
2D & $5.83(1.49)$ & $5.26,6.39$ & 6.00 & 2.219 \\
VR & $5.84(1.59)$ & $5.25,6.42$ & 6.00 & 2.540
\end{tabular}

Table 1. Descriptive statistics of the pretest and posttest positive and negative affect scores in all three conditions.

A significant correlation found between cued recall (Memory) and the baseline negative affect score ( pre NA) called for prudence as different baseline scores in treatment samples could cause a misguided interpretation of the overall results when comparing those groups. However, the Kruskal-Wallis test used for comparing independent samples of multiple groups suggested no difference in the baseline NA between the treatment groups $H(2)=1.18, p=.554$.

\begin{tabular}{|c|c|c|c|c|c|c|c|c|c|}
\hline \multicolumn{10}{|c|}{ Correlation Matrix } \\
\hline \multicolumn{2}{|c|}{ Variable } & (1) & (2) & (3) & (4) & (5) & (6) & (7) & (8) \\
\hline (1) & Pre PA & 1.000 & & & & & & & \\
\hline (2) & Post PA & $.627 * *$ & 1.000 & & & & & & \\
\hline (3) & Pre NA & .076 & -.009 & 1.000 & & & & & \\
\hline (4) & Post NA & $.226^{*}$ & .044 & $.627 * *$ & 1.000 & & & & \\
\hline (5) & PA Change & $-.398 * *$ & $.465^{* *}$ & -.097 & -.205 & 1.000 & & & \\
\hline (6) & NA Change & .198 & .063 & $-.286^{* *}$ & $.567 * *$ & -.150 & 1.000 & & \\
\hline (7) & Age & .064 & -.028 & -.210 & -.200 & -.106 & -.024 & 1.000 & \\
\hline (8) & Memory & -.001 & .020 & $-.261 *$ & $-.219 *$ & .025 & .006 & .080 & 1.000 \\
\hline
\end{tabular}

Table 2. The correlation matrix of all variables.

\subsection{Emotional response}

The conditions' effects on participants' emotional states were investigated in two separate ways. Firstly, the direct effects of conditions on positive and negative affect changes were tested using the Pre and Post pairs of variables for both PA and NA. In this case, due to non-normal distribution of NA, Wilcoxon Signed-Rank test was used to determine whether single conditions evoked changes in the emotional responses. Secondly, the independent samples Kruskal-Wallis test was used to assess whether the change in emotional states differed between the experiment groups. For the latter, the used variables were PA Change and NA Change. Boxplots of all of the relevant variables are presented in Figure 2 and Figure 3.

\begin{tabular}{lccccccccc} 
& \multicolumn{3}{c}{ Positive Affect Change } & & \multicolumn{4}{c}{ Negative Affect Change } \\
\cline { 2 - 7 } \cline { 7 - 10 } Condition & $p$ & $Z$ & $r$ & $r^{2}$ & & $p$ & $Z$ & $r$ & $r^{2}$ \\
\hline Article & $\mathbf{. 0 0 2}$ & -3.05 & .42 & .17 & & $\mathbf{. 0 1 1}$ & -2.55 & .35 & .12 \\
2D & $\mathbf{. 0 1 0}$ & -2.58 & .34 & .11 & & $\mathbf{. 0 0 1}$ & -3.34 & .44 & .19 \\
VR & $\mathbf{. 0 1 5}$ & -2.44 & .19 & .04 & & $<.001$ & -.3 .96 & .50 & .25 \\
\hline
\end{tabular}

Table 3. Wilcoxon Signed-Rank test results on the positive and negative affect changes in individual conditions. 
Note. $\mathrm{r}$ is the effect size calculated as $\mathrm{Z} / \mathrm{sqrt}(\mathrm{N}) ; \mathrm{r}^{2}$ presents the percent of variance explained by the treatment (e.g. .17 is $17 \%$ of variance).

Paired samples tests indicated that likely all three conditions affected both positive and negative affect states (Table 3), supporting H1.1 and H1.2. When it comes to the differences between conditions' effects on PA, the test suggested to retain the null hypothesis $(H(2)=.195, p=.907$, etasq $=.02$ ), or that conditions elicited similar levels of change in the positive affect, leading to no support for H2.1. However, the test for negative affect indicated there are differences between the treatments' effects on the negative affect change $(H(2)=7.00, p=.030$, etasq $=.06)$. The results of the Dunn's test for the post-hoc pairwise comparisons of the negative affect partially supported H2.2 and are reported in Table 4.

\begin{tabular}{lccc} 
& \multicolumn{3}{c}{ Negative affect across treatments } \\
\cline { 2 - 4 } Treatment pair & $Z$ & $p$ & $p_{a d j}$ \\
\hline Article - 2D & 2.10 & .036 & .108 \\
Article - VR & 2.48 & .013 & $\mathbf{. 0 4 0}$ \\
2D - VR & -.35 & .725 & 1.000
\end{tabular}

Table 4. Dunn's test results on pairwise comparisons of negative affect changes.

Note. $p_{a d}$ is the corrected $p$ value using the Bonferroni method.

It is important to note that the distribution of the NA change in immersive conditions has a noticeably higher variance than the article condition. This resulted in that, for example, approximately $25 \%$ of those in this study's sample designated to the $2 \mathrm{D}$ condition had a distinctly and uniquely higher negative response than those who read the Article (Figure 2).

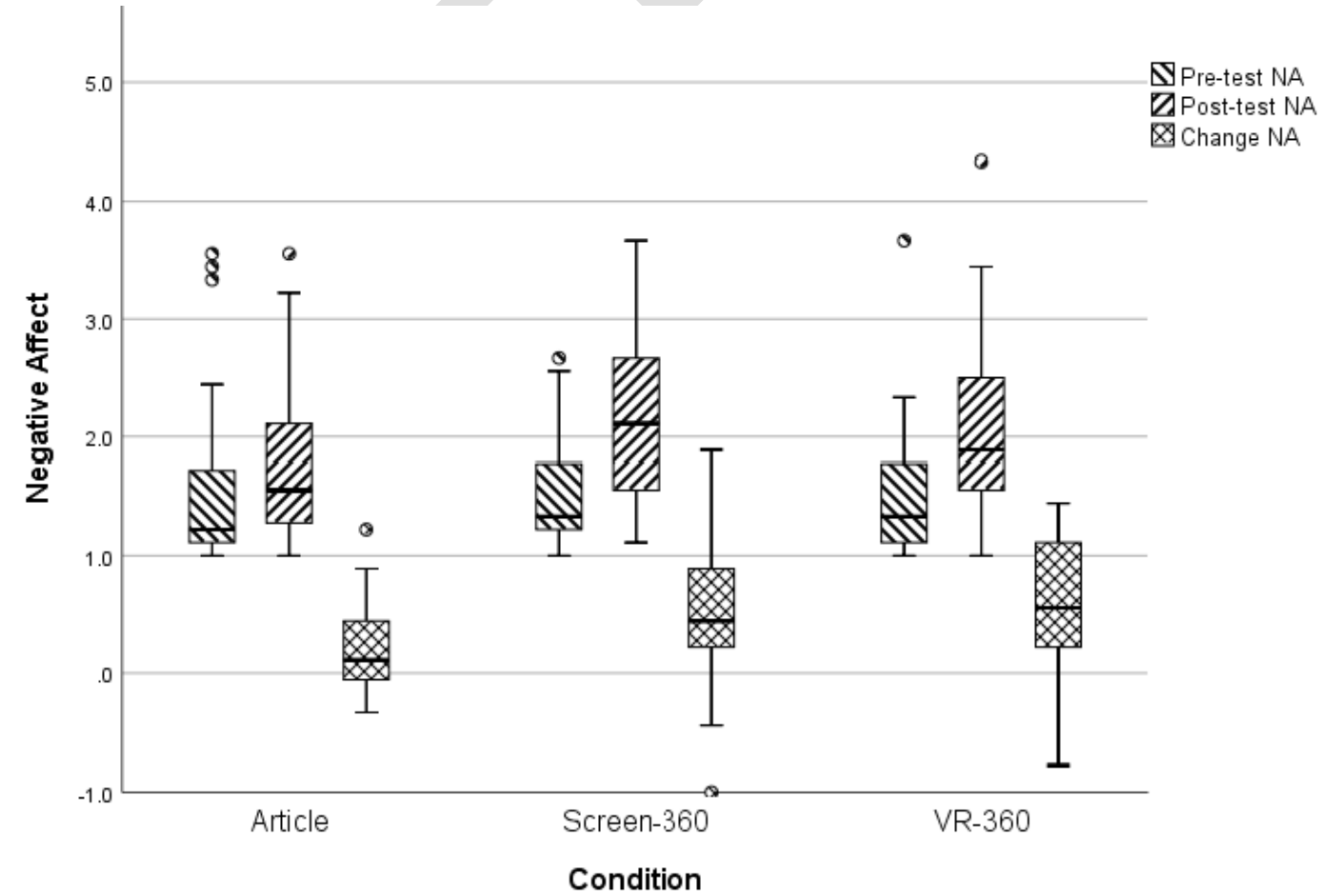

Figure 2. Boxplot chart of the pre-test, post-test, and change scores of Negative Affect in the three conditions. 
Additionally, when looking into the effects of gender on the negative emotional response, H3.1 suggested that females $(\mathrm{Mdn}=.722)$ would report a higher negative emotional change than males $(\mathrm{Mdn}=.333)$. This hypothesis was supported by the results of a Mann Whitney $\mathrm{U}$ test, $p=$ .014 . The effect size was computed as $r=.27$, meaning that $7.3 \%$ of variance in the negative affect change can be explained by the user's reported gender.

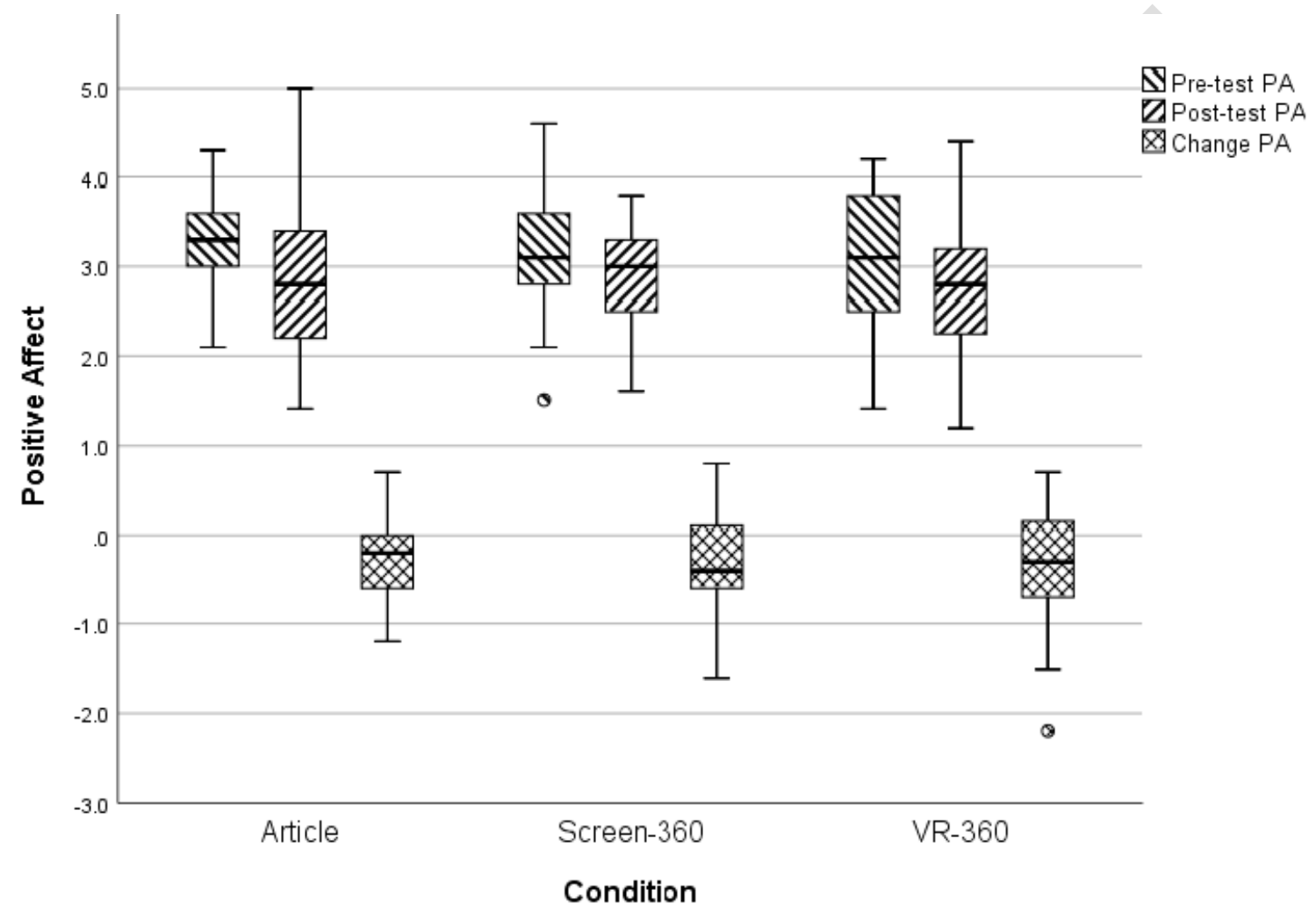

Figure 3. Boxplot chart of the pre-test, post-test, and change scores of Positive Affect in the three conditions.

\subsection{Cued Recall}

A representation of the frequency of the total number of correct answers for all three conditions is presented using boxplots (Figure 4). Although there is no apparent average difference between the groups, distributions are very different between the immersive conditions (2D and VR) compared to the Article condition. The visualisation suggests that higher scores were more frequent in the Article condition, with only four outliers scoring below 5 points, whereas $25 \%$ of those in the 2D condition scored between 2 and 5 points. On the other hand, inference testing using Kruskal-Wallis suggests that the $\mathbf{H 4 . 2}$ is not supported $(H(2)=2.35, p=.309$, etasq $=$ .004). For a comprehensive interpretation, it should be emphasized that the cued recall was tested using a single variable representing the sum of correct answers totaling an integer scale from 0 to 10 , which by itself does not provide a lot of granularity to the data and might not be able to show small effects on variance that are apparent from the boxplot chart. 


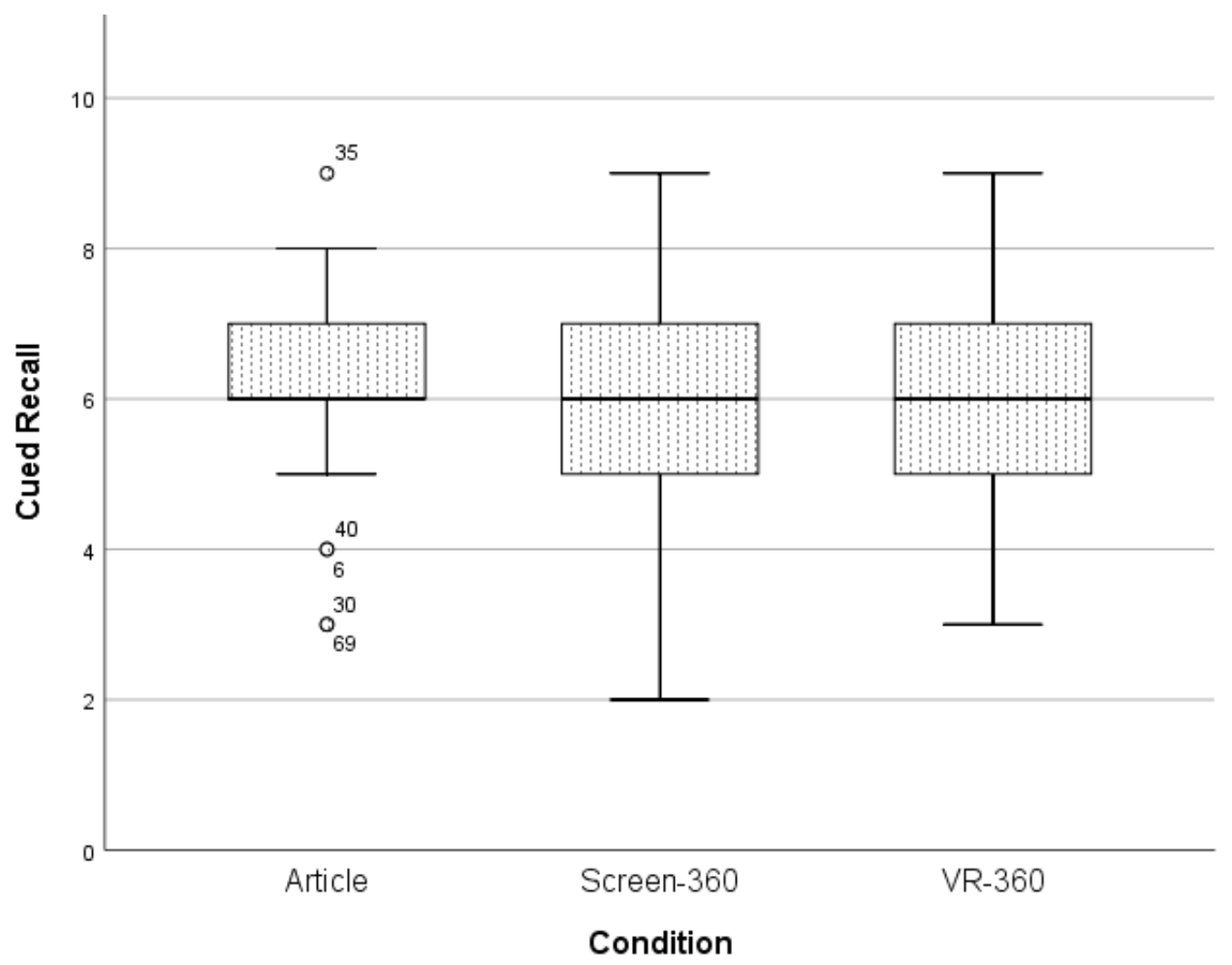

Figure 4. Boxplot chart of Cued Recall Sum scores in the three conditions.

Finally, when assessing the effects of the negative affect state on the cued recall, a simple linear regression was found statistically significant $\left(F(1,85)=4.28, p=.042, \mathrm{R}^{2}=.048\right)$. On average, a full point of post-test negative affect post decreases the correct answers by $.446(\beta=-.22)$, supporting H4.1. However, the scatterplot of the relationship between negative affect and cued recall (Figure 5) does not seem to present clear effects. When considering the statistical inference test and the accompanying plot, it might be worth noting that the cued recall is on an ordinal scale rather than continuous, limiting the granularity of data representation and analysis. 


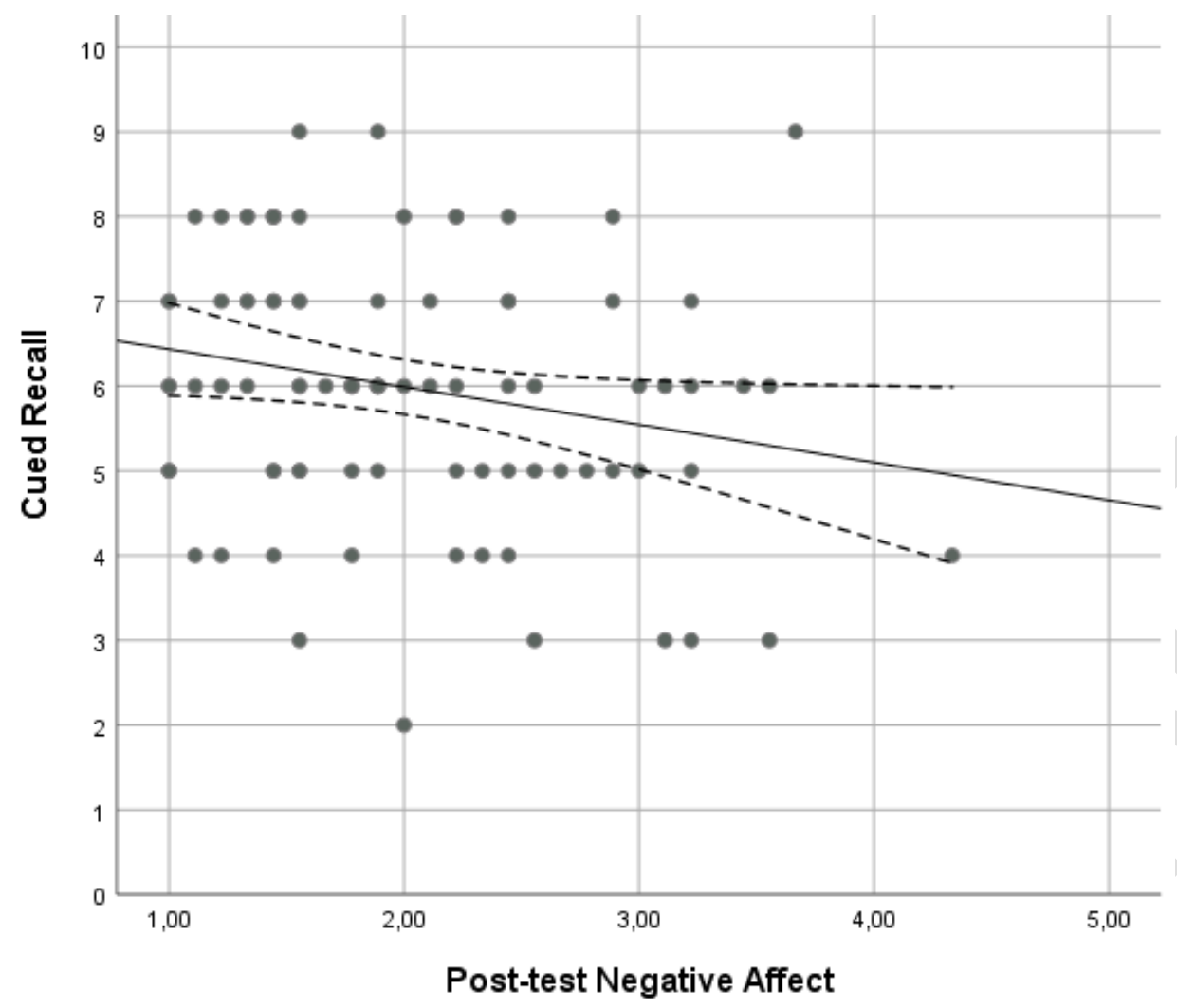

Figure 5. Scatterplot showing the relationship between Cued Recall sum score and Post-test Negative Affect with a 95\% Confidence Interval.

\subsection{Results summary}

For an approachable overview, all hypotheses, related tests, and the outcomes are recapitulated and presented in Table 5.

\section{Hypothesis}

Result

$\operatorname{Test}(\mathrm{s})$

Consuming first-person media

H1. 1 about someone's hardships elicits a lowered positive affect regardless of the media type.

Consuming first-person media

H1.2 about someone's hardships elicit a heightened negative affect regardless of the media type.

Higher immersiveness of media $\mathrm{H} 2.1$ representing someone's hardships predicts a higher decrease in the positive affect.
Supported

Wilcoxon Signed-

Rank

Supported

Wilcoxon Signed-

Rank

Not supported Kruskal-Wallis H 


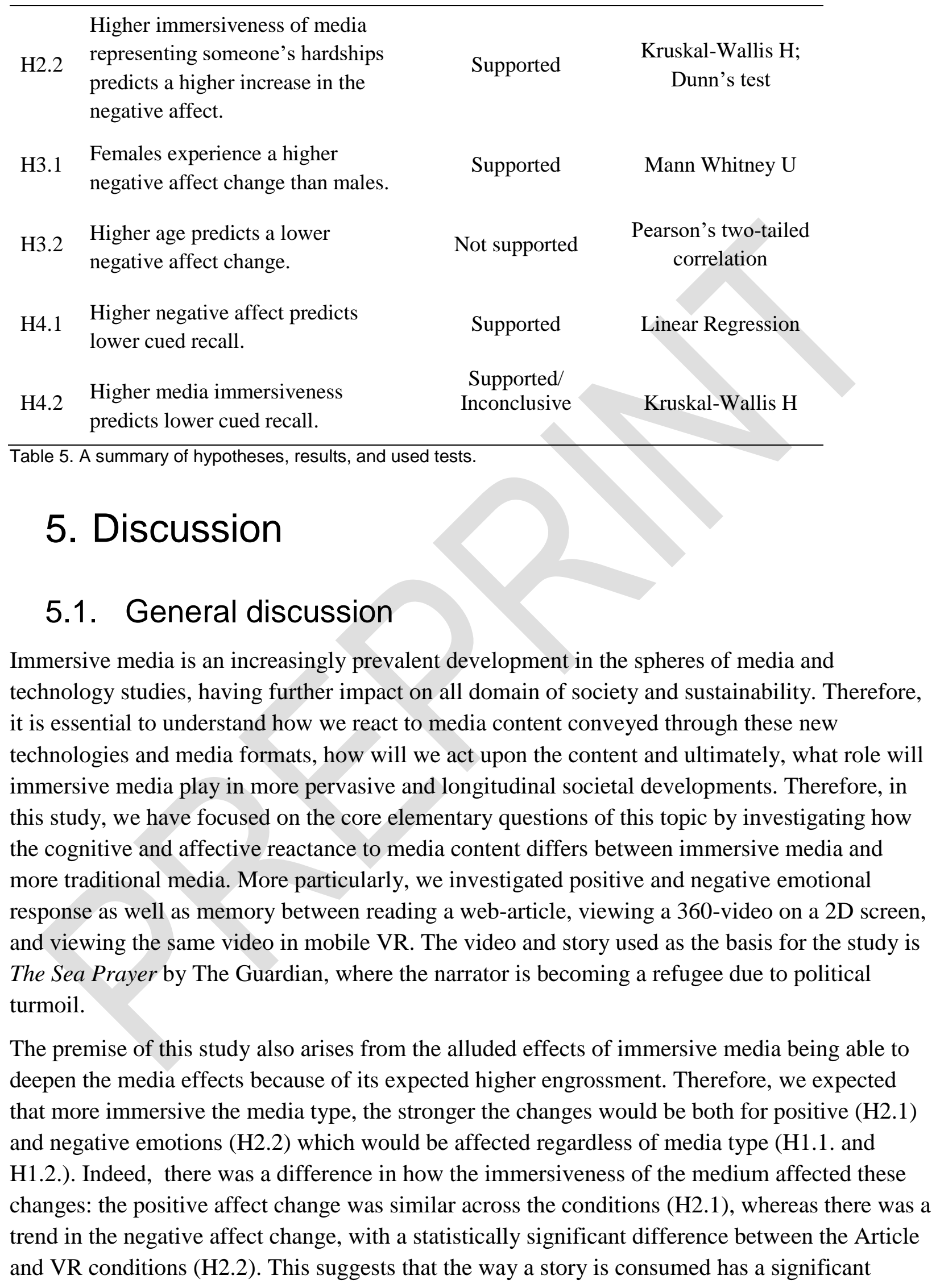


impact on users' emotional responses. This is further supported by the effect sizes which indicate not only statistically significant but also non-trivial effects.

Aside from emotional reaction, an overall media experience also include cognitive aspects, or what and how accurate the consumer can remember from the content. There was no statistically significant difference in how the conditions affected cued recall. However, cued recall distribution variance is noticeably different between 2D and VR on one side and Article on the other. Unlike the affect change, the memory scores were on an integer 1-10 scale, which to an extent impairs a nuanced comparison. Regardless, it would seem that users' cued recall is similar regardless of how the story was consumed, with a trend of a greater number of low scores in the immersive conditions. Furthermore, it might very well be so that the immersive journalism conditions combined do differ from article in terms of memory.

When it comes to the relationship between the negative affect and memory, this study suggests a possible link between the negative affect state after the treatment and memory across the conditions. This relationship does not appear to be trivial, but the magnitude of the effect suggests that the negative affect would need to be heightened further than it has during the experiment so as to have real-world consequences on users' recall of the information presented. In other words, the emotional effects of a similar single immersive journalism 360-degree video likely would not be noticeably detrimental to remembering factual information presented in the one video. These results are in line with previous ones on verbatim memory and negative valence (Van Damme and Smets 2014, Brainerd et al. 2010) as well with those by Cadet and Chainay (2020) who investigated these effects in immersive environments. In the latter, the authors could not explain their results as they relied on the emotional enhancement theory of memory which supposes that stronger emotions enhance memory, regardless of valence. However, the combined results from this study and the one by Cadet and Chainay (2020) might indicate that heightened negative valence in immersive surroundings impedes verbatim memory. This seems to hold true both for cued and free recall and should especially be considered in real-world scenarios such as repeated or prolonged exposure to similar stimuli, which might have higher effects on one's negative affect and consequently factual memory of the materials.

Finally, participants' age did not seem to have any effect on the results, but gender did. It would seem that those identifying as females would report on average about 0.4 higher negative affect change than those identifying as males, on a scale from 1 to 7 . In terms of memory, it would translate to around 0.2 points difference on a 1-10 scale. Therefore, in the scenario of viewing a single 360-degree video, even though the difference in the emotional response is not trivial and possibly has effects on other outcomes, memory does not seem to be affected by age and gender in real-world terms. However, it should be considered that there are other moderators of the effect of age, relevant to the content of the stimulus. For example, as the presented story revolves around protecting a child, the emotional response can be influenced by whether participants have experienced parenthood or not. 


\subsection{Practical and theoretical implications}

Although the stimulus used here was a 360-degree video inspired by true events and reflecting a real global concern implicating whole societies, the practical implications of the results are multifold and applicable to a variety of areas and types of immersive applications. In that vein, this study contributes to the overall fields of media studies, human-computer interaction, and media psychology.

When it comes specifically to immersive journalism, it is primarily worth considering that, in laboratory conditions, there exists a trend of somewhat lower memory of immersive journalism contents than of written article. Due to this being one of the first studies on the topic, all possible indications of undesirable effects should be taken into account before they are investigated further and strengthened or rejected. The difference found in this study indeed does seem small, but repeated or prolonged exposure might further exacerbate these effects, while an improved immersive media literacy might dampen the strength of the effects of an individual experience.

On the other hand, literature on emotions and memory suggests that there is an apparent benefit in highly affective media when it comes to remembering the experience and the gist, meaningbased content (Bower, Kark and Kensinger 2017). The aims of the production should then be carefully considered and the emotional aspect of the immersive media readjusted in order to balance potential benefits such as the feeling of presence, or being-there, (Sundar, Kang and Oprean 2017, Shin and Biocca 2018), following up on the topic (e.g. Steed et al. 2018), and attitudinal changes (e.g. Bujić et al. 2020, Herrera et al. 2018) against the potential ramifications of the experience, such as reduced memory of the presented facts and even construction of false memories (Brainerd et al. 2008). These issues should be considered in particular when accounting for the negativity bias hypothesis which explains people's draw towards consuming negative news (e.g. Soroka, Fournier and Nir 2019). Careless hyper-production of such content might drive views and hyper-consumption, but at the same time could greatly contribute to spreading misinformation due to impaired recall of the content.

These implications based on immersive journalism content extend far beyond to virtually all spheres of immersive media applications that might need to consider the outcomes related to memory more strictly. When it comes to education, for example, these effects on emotions and memory should be considered when choosing the appropriate media for delivering the materials. If the content is neutral or positive, it is likely more suitable to use higher immersion as it has the potential of enhancing emotions and learning (Parong and Mayer 2018, Chirico and Gaggioli 2019). The heightened emotional response has been found in immersive versus desktop video game playing (Pallavicini, Pepe and Minissi 2019), which implies that the effect, as well as consequent effects on memory and learning, should be accounted for in immersive gamified applications and educational games. As these types of interactive and highly engaging immersive experiences are also often used in simulation trainings and other serious applications such as psychotherapy and psychological well-being applications, this relation between affective and cognitive effects should considered for the production of a vast array of immersive experiences. 
Furthermore, theoretical implications derived from the study span primarily in two domains - the relationship of immersive technologies and its affective and cognitive outcomes; and the relationship between emotions and memory. Technological immersiveness has been shown to contribute to a myriad of outcomes relevant for many domains, such as presence (Cummings and Bailenson 2016), attitudes (e.g. Harrera et al. 2018), and intention to continue use (Bujić and Hamari 2020). Additionally, it has been established that immersive media can, in a targeted manner, influence emotions (Felnhofer et al. 2015). These results further build on this understanding of the effects of immersiveness on emotional valence and memory. They contribute to the literature by suggesting that there is a positive relation between the immersiveness of the mediated experience and the resulting emotional responses. In particular, it suggests that even in the context of a 360-degree video content, a simple mobile VR is arguably much more effective at evoking a stronger emotional response, congruent with the valence of the content, than when presented in the form of an article. Furthermore, it considers the moderating effects of gender and age and suggests that the outcomes might be stronger for females than males, as well as that the age differences in a group of 18 to 40-year olds does not affect the emotional experience of immersive media.

Additionally, when considering the technological immersiveness and memory, there seems to be somewhat unreliable difference in how different immersive media affect cued recall of the content. However, further research is warranted as there might be a slight trend suggesting better memory when reading an article than watching a 360-degree video on screen or in mobile VR. This can be explained by the identified negative relationship between the negative affect state and memory when considered together with the media effects on the negative affect. It is possible that the effects of the treatment on emotions, and of emotions on memory both might be small or varied enough not to show a definitive connection of treatments and memory. Additionally, it has been suggested that immersive environments can lead to the overload of cognitive capacities, impeding memory (Bailey et al. 2012). Previous research in educational and simulation contexts has usually considered neutral and positive stimuli (e.g. Chittaro and Buttussi 2015), as opposed to negative, which might mislead into simplified understanding that immersiveness could in itself enhance memory.

\subsection{Limitations and further studies}

The primary limitation of the current study is its focus only on the type of stimulus that induces higher negative valence and with an exploratory approach to novel topics, hence using a single base stimulus in three conditions. Although this is in line with the wider context of immersive journalism where usually distressing and overall negative topics would be in focus, positive images are also relevant for the broader context of understanding the relation of immersive media, emotions, and memory. Additionally, as both arousal and valence play important roles in how stimuli are perceived and remembered, future research should also consider utilising 
factorial study designs with stimuli that evoke different levels of both arousal and negative valence.

Due to the lack of understanding of the differences of mechanism between emotions on one side and gist and verbatim memory on the other (Bower, Kark, and Kensinger 2017), a more nuanced investigation of the effects of immersive media on both of these types of memory is needed. The results in this study consider the overall cued recall of the information presented through different mean as words or images. This study contributes to our understanding of remembering of similar immersive journalism content and calls for following ones to consider how to better understand the causes and mechanisms behind the results, consequently providing guidance for media producers.

An additional potential line of inquiry for future studies of the effects of immersive virtual reality applications that aim at evoking the subjective feeling of taking part as an actor in the depicted events is autobiographical memory (Williams, Conway, and Cohen 2008). In contrast to episodic memories, autobiographical memories have a shorter retrieval time and are closely integrated with the sense of self and related to other autobiographical memories. Thus far, there is an indication that IVR experiences may be powerful enough to be associated with the autobiographical associative network (Schöne, Wessels and Gruber 2019), with possible farreaching repercussions on the implications of IVR, such as attitudes and behaviour.

Another understudied consideration in the context of immersive VR and immersive journalism are the implications of gender and individual differences on affective responses and memory. For example, neuroticism as one of the Big Five personality traits that depicts susceptibility to negative emotions, could enhance the negative valence after viewing an emotional story. Identifying these moderators of the effects of media would lead to better understanding and managing of appropriate modes of delivering content depending on the intended audience. Similarly, age might be a relevant factor as there seems to exist a positivity effect, as an opposite to the negativity bias, in older age (Cartensen and DeLiema 2018, Wood and Kisley 2006). The individual differences that were considered in this study are only secondary, examining examples of possible factors that moderate the main effects of emotions on memory. This is partially also due to the particular participant sample composition that was accessible for this research, consisting mostly of quite young students with the average age of 24.6 years.

Finally, and most importantly, this study contributes by complementing the vast previous literature that considers the effects of emotions on memory by investigating a novel real-world context. Upcoming studies in immersive media should carefully consider what explanatory mechanisms might be the most relevant for effective and ethical future of immersive journalism and related applications of VR. 


\section{Acknowledments}

This study was funded by Business Finland as a part of project Immersive (5479/31/ 2017) and Foundation for Economic Education as a part of project GamEmOrg (16-9394).

\section{REFERENCES}

Baía Reis, A., \& Coelho, A. F. V. C. C. (2018). Virtual Reality and Journalism: A gateway to conceptualizing immersive journalism. Digital Journalism, 6(8), 1090-1100.

Bailenson, J. (2018). Experience on demand: What virtual reality is, how it works, and what it can do. WW Norton \& Company.

Bailey, J., Bailenson, J. N., Won, A. S., Flora, J., \& Armel, K. C. (2012, October). Presence and memory: immersive virtual reality effects on cued recall. In Proceedings of the International Society for Presence Research Annual Conference (pp. 24-26).

Birditt, K. S. (2014). Age differences in emotional reactions to daily negative social encounters. Journals of Gerontology Series B: Psychological Sciences and Social Sciences, 69(4), 557-566.

Bogost, I. (2007). Persuasive games (Vol. 5). Cambridge, MA: MIT Press.

Bolls, P. D., Lang, A., \& Potter, R. F. (2001). The effects of message valence and listener arousal on attention, memory, and facial muscular responses to radio advertisements. Communication Research, 28(5), 627-651.

Bookbinder, S. H., \& Brainerd, C. J. (2017). Emotionally negative pictures enhance gist memory. Emotion, 17(1), 102.

Bowen, H. J., Kark, S. M., \& Kensinger, E. A. (2018). NEVER forget: negative emotional valence enhances recapitulation. Psychonomic Bulletin \& Review, 25(3), 870-891

Brainerd, C. J., Stein, L. M., Silveira, R. A., Rohenkohl, G., \& Reyna, V. F. (2008). How does negative emotion cause false memories?. Psychological science, 19(9), 919-925.

Brainerd, C. J., \& Reyna, V. F. (2004). Fuzzy-trace theory and memory development. Developmental Review, 24(4), 396-439.

Bujić, M., \& Hamari, J. (2020a). Satisfaction and willingness to consume immersive journalism: experiment of differences between VR, 360 video, and article. In Proceedings of the 23rd International Conference on Academic Mindtrek (pp. 120-125).

Bujić, M., \& Hamari, J. (2020b). Immersive journalism: Extant corpus and future agenda. In GamiFIN Conference 2020: Proceedings of the 4th International GamiFIN Conference (pp. 136-145). in GamiFIN Conference 2020. 
Bujić, M., Salminen, M., Macey, J., \& Hamari, J. (2020). "Empathy machine”: how virtual reality affects human rights attitudes. Internet Research.

Cadet, L. B., \& Chainay, H. (2020). Memory of virtual experiences: Role of immersion, emotion and sense of presence. International Journal of Human-Computer Studies, 144, 102506.

Carstensen, L. L., \& DeLiema, M. (2018). The positivity effect: A negativity bias in youth fades with age. Current opinion in behavioral sciences, 19, 7-12.

Chirico, A., \& Gaggioli, A. (2019). When virtual feels real: comparing emotional responses and presence in virtual and natural environments. Cyberpsychology, Behavior, and Social Networking, 22(3), 220-226.

Chittaro, L., \& Buttussi, F. (2015). Assessing knowledge retention of an immersive serious game vs. a traditional education method in aviation safety. IEEE transactions on visualization and computer graphics, 21(4), 529-538.

Crawford, J. R., \& Henry, J. D. (2004). The Positive and Negative Affect Schedule (PANAS): Construct validity, measurement properties and normative data in a large non-clinical sample. British journal of clinical psychology, 43(3), 245-265.

Cuff, B. M., Brown, S. J., Taylor, L., \& Howat, D. J. (2016). Empathy: A review of the concept. Emotion review, 8(2), 144-153.

Cummings, J. J., \& Bailenson, J. N. (2016). How immersive is enough? A meta-analysis of the effect of immersive technology on user presence. Media Psychology, 19(2), 272-309.

De la Peña, N., Weil, P., Llobera, J., Giannopoulos, E., Pomés, A., Spanlang, B., ... \& Slater, M. (2010). Immersive journalism: immersive virtual reality for the first-person experience of news. Presence: Teleoperators and virtual environments, 19(4), 291-301.

De Vignemont, F., \& Singer, T. (2006). The empathic brain: how, when and why?. Trends in cognitive sciences, 10(10), 435-441.

Ding, N., Zhou, W., \& Fung, A. Y. (2018). Emotional effect of cinematic VR compared with traditional 2D film. Telematics and Informatics, 35(6), 1572-1579.

Doherty, R. W., Orimoto, L., Singelis, T. M., Hatfield, E., \& Hebb, J. (1995). Emotional contagion: Gender and occupational differences. Psychology of Women Quarterly, 19(3), 355-371.

Dibben, N. (2002). Gender identity and music. Musical identities, 117-133.

Elfenbein, H. A. (2014). The many faces of emotional contagion: An affective process theory of affective linkage. Organizational Psychology Review, 4(4), 326-362.

Felnhofer, A., Kothgassner, O. D., Schmidt, M., Heinzle, A. K., Beutl, L., Hlavacs, H., \& Kryspin-Exner, I. (2015). Is virtual reality emotionally arousing? Investigating five emotion inducing virtual park scenarios. International journal of human-computer studies, $82,48-56$. 
Gomes, C. F., Brainerd, C. J., \& Stein, L. M. (2013). Effects of emotional valence and arousal on recollective and nonrecollective recall. Journal of Experimental Psychology: Learning, Memory, and Cognition, 39(3), 663.

Herrera, F., Bailenson, J., Weisz, E., Ogle, E., \& Zaki, J. (2018). Building long-term empathy: A large-scale comparison of traditional and virtual reality perspective-taking. PloS one, 13(10), e0204494.

Jacobs, R. S. (2020). Serious games: Play for change. In The Video Game Debate 2 (pp. 19-40). Routledge.

Kensinger, E. A. (2009). Remembering the details: Effects of emotion. Emotion Review, 1(2), 99-113.

Kensinger, E. A., \& Corkin, S. (2003). Memory enhancement for emotional words: Are emotional words more vividly remembered than neutral words?. Memory \& cognition, 31(8), 1169-1180.

Kevrekidis, P., Skapinakis, P., Damigos, D., \& Mavreas, V. (2008). Adaptation of the Emotional Contagion Scale (ECS) and gender differences within the Greek cultural context. Annals of general psychiatry, 7(1), 14.

Koivisto, J., \& Hamari, J. (2019). The rise of motivational information systems: A review of gamification research. International Journal of Information Management, 45, 191-210.

Kim, A., Chang, M., Choi, Y., Jeon, S., \& Lee, K. (2018, March). The effect of immersion on emotional responses to film viewing in a virtual environment. In 2018 IEEE Conference on Virtual Reality and 3D User Interfaces (VR) (pp. 601-602). IEEE.

Kool, H. (2016). The Ethics of Immersive Journalism: A rhetorical analysis of news storytelling with virtual reality technology. Intersect: the stanford journal of science, technology, and society, 9(3).

Lakens, D. (2021). Sample size justification. PsyArXiv, doi: 10.31234/osf.io/9d3yf.

Lang, A. (2000). The limited capacity model of mediated message processing. Journal of Communication, 50, 46-70.

Lang, A., Dhillon, K., \& Dong, Q. (1995). The effects of emotional arousal and valence on television viewers' cognitive capacity and memory. Journal of Broadcasting \& Electronic Media, 39(3), 313-327.

Martel, C., Pennycook, G., \& Rand, D. G. (2020). Reliance on emotion promotes belief in fake news. Cognitive research: principles and implications, 5(1), 1-20.

Matthes, J. (2013). The affective underpinnings of hostile media perceptions: Exploring the distinct effects of affective and cognitive involvement. Communication Research, 40(3), 360-387.

Pallavicini, F., Pepe, A., \& Minissi, M. E. (2019). Gaming in virtual reality: What changes in terms of usability, emotional response and sense of presence compared to non-immersive video games?. Simulation \& Gaming, 50(2), 136-159. 
Parong, J., \& Mayer, R. E. (2018). Learning science in immersive virtual reality. Journal of Educational Psychology, 110(6), 785.

Potter, W. J. (2010). The state of media literacy. Journal of broadcasting \& electronic media, 54(4), 675-696.

Raessens, J. (2019). Virtually Present, Physically Invisible: Alejandro G. Iñárritu's Mixed Reality Installation Carne y Arena. Television \& New Media, 20(6), 634-648.

Reyna, V. F., Corbin, J. C., Weldon, R. B., \& Brainerd, C. J. (2016). How fuzzy-trace theory predicts true and false memories for words, sentences, and narratives. Journal of applied research in memory and cognition, 5(1), 1-9.

Robinson-Riegler, G. L., \& Winton, W. M. (1996). The role of conscious recollection in recognition of affective material: Evidence for positive-negative asymmetry. The Journal of General Psychology, 123(2), 93-104.

Salminen, M., Järvelä, S., Ruonala, A., Timonen, J., Mannermaa, K., Ravaja, N., \& Jacucci, G. (2018, March). Bio-adaptive social VR to evoke affective interdependence: DYNECOM. In 23rd international conference on intelligent user interfaces (pp. 73-77).

Sánchez Laws, A. L. (2020). Can immersive journalism enhance empathy?. Digital Journalism, 8(2), 213-228.

Sánchez Laws, A. L., \& Utne, T. (2019). Ethics guidelines for immersive journalism. Frontiers in Robotics and AI, 6, 28.

Shin, D. (2018). Empathy and embodied experience in virtual environment: To what extent can virtual reality stimulate empathy and embodied experience?. Computers in Human Behavior, 78, 64-73.

Shin, D., \& Biocca, F. (2018). Exploring immersive experience in journalism. New media \& society, 20(8), 2800-2823.

Schöne, B., Wessels, M., \& Gruber, T. (2019). Experiences in virtual reality: A window to autobiographical memory. Current Psychology, 38(3), 715-719.

Soroka, S., Fournier, P., \& Nir, L. (2019). Cross-national evidence of a negativity bias in psychophysiological reactions to news. Proceedings of the National Academy of Sciences, 116(38), 18888-18892.

Spaniol, J., Voss, A., \& Grady, C. L. (2008). Aging and emotional memory: Cognitive mechanisms underlying the positivity effect. Psychology and Aging, 23(4), 859-872.

Steed, A., Pan, Y., Watson, Z., \& Slater, M. (2018). "We wait"-the impact of character responsiveness and self embodiment on presence and interest in an immersive news experience. Frontiers in Robotics and AI, 5, 112.

Sundar, S. S., Kang, J., \& Oprean, D. (2017). Being there in the midst of the story: How immersive journalism affects our perceptions and cognitions. Cyberpsychology, Behavior, and Social Networking, 20(11), 672-682. 
Swaminathan, S., \& Schellenberg, E. G. (2015). Current emotion research in music psychology. Emotion review, 7(2), 189-197.

Valkenburg, P. M., Peter, J., \& Walther, J. B. (2016). Media effects: Theory and research. Annual review of psychology, 67, 315-338.

Van Damme, I., \& Smets, K. (2014). The power of emotion versus the power of suggestion: Memory for emotional events in the misinformation paradigm. Emotion, 14(2), 310.

Visch, V. T., Tan, E. S., \& Molenaar, D. (2010). The emotional and cognitive effect of immersion in film viewing. Cognition and Emotion, 24(8), 1439-1445. H2.1: Higher immersiveness of media representing someone's hardships predicts a higher decrease in the positive affect.

Watson, D., Clark, L. A., \& Tellegen, A. (1988). Development and validation of brief measures of positive and negative affect: the PANAS scales. Journal of personality and social psychology, 54(6), 1063.

Williams, H. L., Conway, M. A., \& Cohen, G. (2008). Autobiographical memory. In G. Cohen \& M. A. Conway (Eds.), Memory in the real world (p. 21-90). Psychology Press.

Wilson, G., \& McGill, M. (2018, October). Violent video games in virtual reality: Re-evaluating the impact and rating of interactive experiences. In Proceedings of the 2018 Annual Symposium on Computer-Human Interaction in Play (pp. 535-548).

Wirth, W., \& Schramm, H. (2005). Media and emotions. Communication Research Trends, 24(3), 3-39. https://www.zora.uzh.ch/id/eprint/101679/1/v24_3.pdf

Wood, S., \& Kisley, M. A. (2006). The negativity bias is eliminated in older adults: Age-related reduction in event-related brain potentials associated with evaluative categorization. Psychology and aging, 21(4), 815.

Yuan, J., Ju, E., Meng, X., Chen, X., Zhu, S., Yang, J., \& Li, H. (2015). Enhanced brain susceptibility to negative stimuli in adolescents: ERP evidences. Frontiers in behavioral neuroscience, $9,98$. 


\section{APPENDIX - CUED RECALL}

Correct answers are marked in bolded lettering.

1. How many visuals scenes were shown?
a. 4
b. 5
c. 3

2. What was the dominant color in the first scene?
a. yellow
b. green
c. purple

3. What was the dominant color in the third scene?
a. green
b. yellow
c. purple

4. What were the colors on the flags in the second scene (regardless of the order)?
a. blue, white, and black
b. black, red, and white
c. white, green, and red

5. Whose childhood memories were first being described?
a. narrator's mother
b. narrator's
c. narrator's son

6. What is the name of the boy that the narrator is talking to?
a. Marwan
b. Muhair
c. Mawhab

7. What is the name of the boy who drowned and served as an inspiration for the story?
a. Aakil
b. Alan
c. Alhan

8. What religious communities are mentioned?
a. only Muslim
b. only Christian
c. Muslim and Christian

9. Where are the actors at the time of narration?
a. In the ruins of their city
b. visiting narrator's old house
c. on a beach

10. How many generations in the narrator's family were mentioned?
a. two
b. three
c. four 